\title{
Nouvelles ressources pour faire face à la résistance aux antibiotiques en quelques clics de souris...
}

\author{
Dowd-Schmidtke C, ${ }^{1 *}$ Tremblay G, ${ }^{1}$ Gale-Rowe M, ${ }^{1}$ Dodds $\mathrm{J},{ }^{1}$ Finley $\mathrm{R}^{2}$
}

${ }^{1}$ Centre de la lutte contre les maladies transmissibles et les infections, Agence de la santé publique du Canada, Ottawa (Ontario)

${ }^{2}$ Centre des maladies infectieuses d'origine alimentaire, environnementale et zoonotique, Agence de la santé publique du Canada, Toronto (Ontario)

* Correspondance à : courtney.dowd-schmidtke@phac-aspc.gc.ca

\section{Résumé}

La résistance aux antibiotiques est un problème complexe dont les causes sont multiples, et il y a de nombreux rôles à jouer pour le résoudre. Dans le cadre de son intervention, l'Agence de la santé publique du Canada est en voie de lancer une campagne pilote de sensibilisation aux antibiotiques pour les familles et les professionnels de la santé canadiens. Coïncidant avec la Semaine de sensibilisation aux antibiotiques qui commence le 17 novembre 2014, l'objectif de cette campagne est d'améliorer les connaissances et la sensibilisation à l'égard de la résistance aux antibiotiques au Canada. Pour y parvenir, l'Agence a élaboré une série de ressources pour les familles et les fournisseurs de soins de santé canadiens, qui comporte une variété de messages clés expliquant la résistance aux antibiotiques, pourquoi c'est important, et comment réduire les risques qui y sont liés. Les ressources pour les familles canadiennes comprennent une vidéo informative en ligne, une brochure éducative, et des infographies pour les adultes et les enfants. Les ressources pour les professionnels de la santé comprennent deux modules de formation médicale continue en ligne, une lettre que les médecins peuvent signer et donner aux parents pour leur expliquer pourquoi un antibiotique n'a pas été prescrit, et deux webinaires pour présenter les tendances en matière de résistance aux antimicrobiens (RAM) et d'utilisation des antimicrobiens. Les professionnels de la santé recevront également une carte postale électronique et une affiche de campagne bilingue. La promotion des messages de la campagne et l'utilisation de ces ressources de campagne soutiendront les professionnels de la santé dans les discussions au sujet de la résistance aux antibiotiques avec leurs patients ou clients, et dans leurs efforts continus visant à faire partie de la solution à ce problème de santé mondial important.

\section{Introduction}

La résistance aux antibiotiques est de plus en plus un problème de santé dans le monde et menace notre capacité à traiter efficacement les maladies et les infections (1). II s'agit d'un problème complexe dont les causes sont multiples, et il y a de nombreux rôles à jouer pour le résoudre. L'Agence de la santé publique du Canada (l'Agence) dirige l'intervention du gouvernement du Canada en réponse au problème de la résistance aux antibiotiques au Canada. Une partie de cette intervention comprend l'élaboration et le lancement d'une campagne pilote de sensibilisation aux antibiotiques pour les familles et les professionnels de la santé canadiens. Le lancement de la campagne pilote de sensibilisation de l'Agence coïncidera avec la Semaine de sensibilisation aux antibiotiques, qui a lieu du 17 au 23 novembre 2014. La Semaine de sensibilisation aux antibiotiques est reconnue au Canada et à l'échelle internationale. Le présent article vise à fournir aux professionnels de la santé des renseignements sur la campagne de l'agence et les ressources connexes.

\section{Objectif, messages clés et ressources}

L'objectif de cette campagne est d'améliorer les connaissances et la sensibilisation en matière de résistance aux antibiotiques au Canada en faisant la promotion de l'utilisation responsable des 
antibiotiques et des bons comportements de prévention des infections. Pour appuyer cet objectif, l'Agence diffusera plusieurs messages clés de promotion de la santé aux Canadiens. Ces messages expliqueront ce qu'est la résistance aux antibiotiques, pourquoi c'est important et comment réduire les risques qui y sont liés. Voici les messages clés :

- La résistance aux antibiotiques est un problème de santé publique.

- Parfois, la meilleure ordonnance, c'est de ne pas en avoir.

- Il y a des mesures faciles à prendre afin de réduire les risques de contracter une infection résistante aux antibiotiques.

- Toujours utiliser les antibiotiques selon la prescription.

Afin d'atteindre ses objectifs, l'Agence a élaboré une série de ressources pour faire face à la résistance aux antibiotiques, destinées aux familles et aux fournisseurs de soins de santé canadiens (2). Les ressources pour les familles canadiennes sont les suivantes : une vidéo informative en ligne; une brochure éducative - Résistance aux antibiotiques : Questions et réponses; deux infographies pour les adultes - Résistance aux antibiotiques : Faits et chiffres, et Contribuer à réduire la résistance aux antibiotiques; ainsi qu'une infographie pour les enfants âgés de 8 à 12 ans intitulée Germes et antibiotiques.

Les ressources à l'intention des professionnels de la santé comprennent deux modules de formation médicale continue en ligne : un sur la résistance aux antimicrobiens (RAM) en général, et un autre précisément sur la résistance aux antimicrobiens et la gonorrhée. Lorsqu'ils seront disponibles, ces modules pourront être consultés sur le site Web d'apprentissage en ligne des Services partagés du gouvernement du Canada et sur les sites Web de diverses associations professionnelles de la santé. Les ressources comprendront également, par exemple, une lettre que les médecins peuvent signer et donner aux parents, leur expliquant pourquoi un antibiotique n'a pas été prescrit pour leur enfant; cette démarche consiste à soutenir davantage l'éducation du grand public à propos de ce problème de santé publique important. En outre, deux webinaires sont prévus en novembre 2014 pour lancer la campagne et présenter les résultats des programmes de surveillance de l'Agence sur les tendances en matière de résistance aux antimicrobiens et d'utilisation des antimicrobiens.

Au cours des prochaines semaines, les professionnels de la santé recevront une carte postale électronique de cartes postales du $D^{r}$ Howard Njoo, directeur général du Centre de la lutte contre les maladies transmissibles et les infections de l'Agence, avec des liens vers ces ressources de campagne sur la résistance aux antibiotiques. Les médecins de famille, les médecins généralistes et les pédiatres canadiens recevront également des copies papier bilingues de l'affiche de la campagne et de la brochure éducative qui peuvent être utilisées pour informer les patients; les pharmacies canadiennes recevront des copies papier de la brochure éducative.

\section{Conclusion}

Le Canada a déjà vu des changements positifs dans les pratiques de prescription visant à freiner la résistance aux antimicrobiens (3). Pour appuyer davantage vos efforts, l'Agence vous invite à consulter le site Web du gouvernement du Canada sur la résistance aux antibiotiques pour en savoir plus sur l'utilisation des antibiotiques et la résistance aux antibiotiques au Canada et pour accéder à nos ressources (2). La promotion des messages de la campagne et l'utilisation de ces ressources de campagne appuieront vos discussions au sujet de la résistance aux antibiotiques avec vos patients ou clients, et vos efforts continus visant à faire partie de la solution à ce problème de santé mondial important. 


\section{Remerciements}

Mille mercis aux collaborateurs de la campagne de sensibilisation, au Centre de la lutte contre les maladies transmissibles et les infections, au Centre des maladies infectieuses d'origine alimentaire, environnementale et zoonotique, à la Direction des communications et des affaires publiques de l'Agence de la santé publique du Canada, à la Direction générale de la santé des Premières nations et des Inuits de Santé Canada et aux membres du groupe consultatif ponctuel pour la campagne de sensibilisation à la résistance aux antimicrobiens.

\section{Conflit d'intérêts}

II n'y a aucun conflit d'intérêts à déclarer.

\section{Financement}

La campagne pilote de sensibilisation a été financée par l'Agence de la santé publique du Canada.

\section{Références}

(1) World Health Organization (WHO). Antimicrobial resistance: Global report on surveillance. Geneva: WHO; 2014. http://apps.who.int/iris/bitstream/10665/112642/1/9789241564748_eng.pdf

(2) Gouvernement du Canada [page Web]. Résistance aux antibiotiques. Mis à jour le 15 octobre 2014. Accès : http://canadiensensante.gc.ca/drugs-products-medicaments-produits/antibiotic-resistanceantibiotique/index-fra.php?_ga=1.87130008.1897391804.1401279223

(3) Agence de la santé publique du Canada. Programme intégré canadien de surveillance de la résistance aux antimicrobiens (PICRA) - Rapport sur l'utilisation des antimicrobiens chez les humains, 2011. Guelph (Ont.) : ASPC: http://www.phac-aspc.gc.ca/cipars-picra/pubs-fra.php 